Article

\title{
A Machine Learning Model Accurately Predicts Ulcerative Colitis Activity at One Year in Patients Treated with Anti-Tumour Necrosis Factor $\alpha$ Agents
}

\author{
Iolanda Valentina Popa ${ }^{1,+}\left(\mathbb{D}\right.$, Alexandru Burlacu ${ }^{1,2,3,+} \mathbb{0}$, Catalina Mihai ${ }^{1,4, *}$ \\ and Cristina Cijevschi Prelipcean ${ }^{4}$ \\ 1 Department of Internal Medicine, University of Medicine and Pharmacy “Gr. T. Popa”, 700115 Iasi, Romania; \\ iolivp@gmail.com (I.V.P.); alburlacu@yahoo.com (A.B.) \\ 2 Head of Department of Interventional Cardiology-Cardiovascular Diseases Institute, 700503 Iasi, Romania \\ 3 Romanian Academy of Medical Sciences, 030167 Bucharest, Romania \\ 4 Institute of Gastroenterology and Hepatology, 700111 Iasi, Romania; cristinacijevschi@yahoo.com \\ * Correspondence: catalinamihai@yahoo.com; Tel.: +40-74-525-8797 \\ + These authors contributed equally to this work.
}

Received: 27 October 2020; Accepted: 18 November 2020; Published: 20 November 2020

check for updates

\begin{abstract}
Background and objectives: The biological treatment is a promising therapeutic option for ulcerative colitis (UC) patients, being able to induce subclinical and long-term remission. However, the relatively high costs and the potential toxicity have led to intense debates over the most appropriate criteria for starting, stopping, and managing biologics in UC. Our aim was to build a machine learning (ML) model for predicting disease activity at one year in UC patients treated with anti-Tumour necrosis factor $\alpha$ agents as a useful tool to assist the clinician in the therapeutic decisions. Materials and Methods: Clinical and biological parameters and the endoscopic Mayo score were collected from 55 UC patients at the baseline and one year follow-up. A neural network model was built using the baseline endoscopic activity and four selected variables as inputs to predict whether a UC patient will have an active or inactive endoscopic disease at one year, under the same therapeutic regimen. Results: The classifier achieved an excellent performance predicting the disease activity at one year with an accuracy of $90 \%$ and area under curve (AUC) of 0.92 on the test set and an accuracy of $100 \%$ and an AUC of 1 on the validation set. Conclusions: Our proposed ML solution may prove to be a useful tool in assisting the clinicians' decisions to increase the dose or switch to other biologic agents after the model's validation on independent, external cohorts of patients.
\end{abstract}

Keywords: inflammatory bowel diseases; artificial intelligence; biological therapy; predictive model; disease activity

\section{Introduction}

Ulcerative colitis (UC) is an inflammatory bowel disease (IBD) with recurrent and remissive evolution. Although the induction of a complete resolution of the disease is not currently possible, UC patients can benefit from the new biological therapies. Anti-Tumor Necrosis Factor $\alpha$ (anti-TNF) agents and other modern biological regimens ensured the success of the "treat to target" approach in UC [1] by their ability to achieve subclinical (endoscopic and histologic) remission [2], corticosteroid therapy discontinuation, reduction in hospitalization and surgery rates, long-term remission, and a good quality of life [3,4].

However, the high costs of biological agents and their potential side effects (mostly related to opportunistic infections and malignancies) [5] have led to intense debates over the most opportune 
timing for starting or discontinuing the therapy, increasing the dose, or switching to another biological regimen and deciding on the most appropriate management of the lack or loss of response [6-8].

Recent studies proved that anti-TNF antibodies are a practical approach for inducing clinical remission in UC [9]. Although promising results, evidence shows that long-term remission is only achieved in a certain proportion of the patients that initially responded to the treatment [10, 11]. Besides, there is insufficient evidence to be sure of the ideal duration of the treatment [12]. The current "status quo" urges further research on optimizing the administration of anti-TNF antibodies. Additional studies for UC patients receiving biological therapy are needed to establish objective criteria for therapeutic decisions.

Modern artificial intelligence/machine learning (ML) solutions may fill this knowledge gap by in-depth investigation of the large clinical datasets available. To date, several innovative ML-based approaches [13] were proposed for various medical topics concerning IBD, such as endoscopic image analysis for the detection of inflammation [14], dysplasia [15], histologic disease activity [16], and disease subtype classification $[17,18]$.

Various studies described ML methods for predicting IBD prognosis. Transcriptomic analyses on purified CD8 T cells and/or whole blood successfully predicted poor prognosis with earlier need for treatment escalation [19]. Other multi-omics ML approaches have been described for predicting IBD treatment outcomes with good performance [20,21]. However, multi-omics techniques are based on costly investigations that are not widely available, making them hardly applicable for routine use. Promising ML solutions for predicting disease outcome after treatment with Vedolizumab [22] or Azathioprine [23] based on standard clinical parameters have been proposed in IBD.

To date, no study based on routinely available clinical data has described ML models for predicting anti-TNF response in UC patients.

We aim to build a machine learning model for predicting disease activity and risk of relapse at one year follow-up in UC patients treated with anti-TNF agents using only standard clinical variables. After the model's validation on independent, external, and sufficiently large cohorts of patients, the proposed ML solution may prove useful in assisting the clinicians' decisions to increase the dose or change the biological agent.

\section{Materials and Methods}

\subsection{Study Design and Participants}

An observational retrospective single-centre cohort study was conducted on a sample of 55 UC patient records. All patients were admitted to the Institute of Gastroenterology and Hepatology, "Sf. Spiridon" Hospital Iași-Romania, between January 2012 and November 2018. Confirmed UC patients under maintenance therapy with an anti-TNF agent (Infliximab/Adalimumab) who underwent a colonoscopy for disease assessment at the initial evaluation and one year follow-up were considered. Only patients in clinical remission at the initial evaluation were included. Patients were excluded if they were in evidence with concurrent disorders (infections, autoimmune and inflammatory conditions, cirrhosis, neoplasia, and hemodialysis) capable of influencing medical parameters, if they presented clinical relapse at the initial evaluation or if changes were made to the therapeutic regimens between the two visits.

All patients provided written informed consent. The study has full ethical approval from the Research Ethics Commission of the "Gr. T. Popa" University of Medicine and Pharmacy (no. 15308/07.2019) and "St. Spiridon" Regional Hospital Ethics Committee (no. 54/10.2019). No sex-based or racial/ethnic-based differences were present.

\subsection{Clinical Protocol}

UC patients were hospitalized for treatment monitoring. According to the European consensus guidelines, UC diagnosis is established by clinical, biochemical, stool, endoscopic, cross-sectional 
imaging, and histological investigations [24]. Patients underwent a medical history interview, physical examination, routine laboratory tests, and colonoscopy to diagnose or assess already diagnosed UC, following the European standard protocols.

All included patients were in clinical remission and under maintenance therapy with an anti-TNF agent: Infliximab (5 mg/kg every eight weeks) or Adalimumab (40 mg every two weeks). No patient received other concomitant immunomodulatory treatment.

\subsection{Data Collection}

The following data were collected both at the initial evaluation and the one year follow-up.

Laboratory parameters documented were: red blood cells (RBC), white blood cells (WBC), platelets (PLT), hemoglobin (HGB), hematocrit (HCT), mean corpuscular hemoglobin concentration (MCHC), plateletcrit (PCT), platelet distribution width (PDW), mean platelet volume (MPV), platelet large cell ratio (PLCR), neutrophils (NEUT), lymphocytes, monocytes (MONO), $\mathrm{C}$ reactive protein (CRP), erythrocyte sedimentation rate/1 h (ESR), fibrinogen, serum iron (SI), ferritin, total proteins (TP), albumin, alpha one globulins (A1G), alpha two globulins (A2G), beta one globulins, beta two globulins, and gamma globulins.

Colonoscopy with biopsy was performed on the EVIS EXERA II endoscopy system (Olympus America). Specialist physicians carried out the procedures from Gastroenterology and Hepatology Institute, Iași, Romania. According to the colonoscopy findings, the Mayo subscore used to classify endoscopic disease activity $[25,26]$ was documented. A patient was considered to have endoscopic remission if the Mayo score was 0 or 1 . Similarly, active disease was considered for Mayo scores 2 or 3 .

\subsection{Preprocessing and Management of Missing Values}

Documented continuous variables (biological parameters) were standardized in the range (0-1). Values of HGB, HCT, SI, and ferritin were processed to resolve the differences between sexes.

Missing values were assigned using multivariate imputation by chained equations (MICE) method implemented by the MICE package in R Studio Version 1.2.1335 (c) 2020-2019 RStudio, Inc. Build 1379 (f1ac3452). Missing variables were assigned by applying the Bayesian regression built-in method.

\subsection{Standard Statistics for Feature Selection}

ANOVA with Holm adjustments in R Studio was used to determine the continuous baseline variables for significant differences between the one year endoscopic remission and active groups. Statistical significance was considered for $p<0.05$. If any two of the selected continuous variables had high intercorrelation with a Pearson coefficient $\geq 0.9$, one of them was removed.

\subsection{Neural Network Model—Construction and Evaluation}

Initial data of $50 \mathrm{UC}$ patient records were randomly divided into a training set of 40 records $(80 \%)$ and a test set of 10 records $(20 \%)$, such that variables distributions in each set were similar to those in the original dataset. The other five patient records from the same medical centre were added independently to be used as a validation set. Endoscopic activity classes (active/inactive) were not equally represented in the train and test set. However, the validation set had a balanced distribution of the disease activity classes.

One multilayered perceptron classifier was developed based upon the training set. The classifier was constructed using the caret: train function in R Studio. A 10-fold cross-validation was used to reduce overfitting. Synthetic minority over-sampling technique (SMOTE) was used with caret: train function to overcome imbalanced data.

The classifier was built to predict whether a UC patient will present endoscopic remission or active disease at the one year follow-up if the therapeutic strategy is left unchanged.

The developed neural network was evaluated on the test set and validation set according to the classification accuracy (ACC). The area under the receiver operating characteristic curve 
(AUC), sensitivity (SE), specificity (SP), positive and negative predictive values (PPV and NPV) were also determined.

\section{Results}

\subsection{Patient Characteristics}

Of all 55 patient records, 40 (72\%) were males and $15(28 \%)$ females. The age range of the participants was 22-62. The distribution of active/inactive endoscopic classes at the one year follow-up was imbalanced: $42 \mathrm{UC}$ patients were reported with endoscopic remission and 13 with active disease.

\subsection{Feature Selection}

Using ANOVA with Holm adjustment, the feature selection step initially included six continuous baseline variables with a significant difference between the active disease group and the remission group at one year: NEUT, PDW, MPV, PLCR, CRP, and A1G. Significance was established at $p<0.05$. Next, highly intercorrelated features were identified and removed. Three strong correlations with a Pearson coefficient $\geq 0.9$ were identified between PDW and MPV, PDW, and PLCR, MPV, and PLCR (Figure 1). Thus, the following parameters were removed from the analysis: MPV and PLCR.

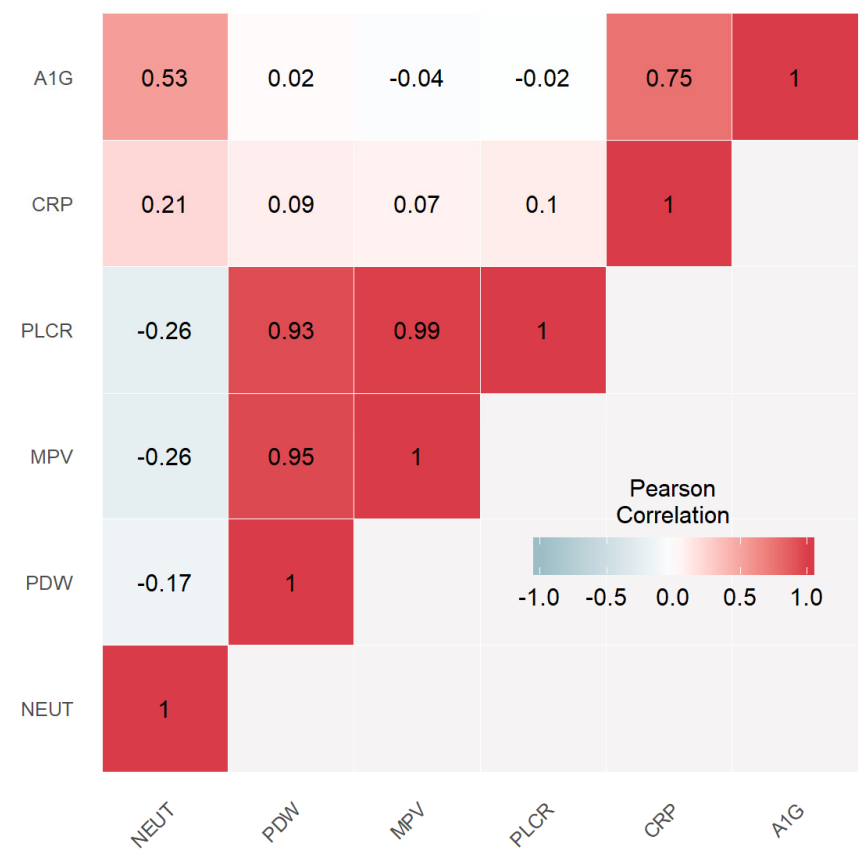

Figure 1. Correlation heatmap showing the Pearson coefficients between all parameters nominated by the feature selection method. NEUT (neutrophils), PDW (platelet distribution width), MPV (mean platelet volume), PLCR (platelet large cell ratio), CRP (C reactive protein), alpha one globulins (A1G).

As a result of the feature selection stage, four parameters (NEUT, PDW, CRP, and A1G) were included in further analysis.

Selected clinical characteristics and laboratory findings for all patient records and each activity class are summarized in Table 1. 
Table 1. Baseline parameters for all patient records and each endoscopic activity class at one year.

\begin{tabular}{cccc}
\hline \multirow{2}{*}{ Baseline Parameters } & \multirow{2}{*}{ All } & \multicolumn{2}{c}{ Endoscopic Activity at One Year } \\
\cline { 3 - 4 } & & Inactive & Active \\
\hline Number of records & 55 & 42 & 13 \\
Gender (male:female) & $40: 15$ & $32: 10$ & $8: 5$ \\
Age (years) & $44.3 \pm 10.5$ & $43.7 \pm 11.4$ & $46 \pm 6.4$ \\
Baseline endoscopic activity & Inactive $\quad 39$ & 35 & 4 \\
NEUT * $10^{3} / \mu \mathrm{L}$ & Active 16 & 7 & 9 \\
PDW fL & $4.59 \pm 2$ & $3.32 \pm 1.13$ & $5.7 \pm 2.37$ \\
CRP mg/dL & $12.9 \pm 1.9$ & $13.2 \pm 2.1$ & $11.8 \pm 1$ \\
A1G $\%$ & $0.35 \pm 0.4$ & $0.3 \pm 0.32$ & $0.55 \pm 0.4$ \\
& $2.1 \pm 0.33$ & $2 \pm 0.32$ & $2.31 \pm 0.27$ \\
\hline
\end{tabular}

* signifies "multiplied by".

\subsection{Handled Missing Values}

UC patient records had a total of 18 (8.2\%) missing values, which were imputed using the MICE package as follows: PDW-4, CRP-2, A1G-12.

\subsection{Results of the Neural Network Models Construction and Evaluation}

Based on the results produced by the feature selection method, a neural network model was trained. The initial dataset of $50 \mathrm{UC}$ patient records was randomly divided into a training set (40 records) and a test set (10 records) to build the classifier. Five patient records were added independently to constitute the validation set. Unlike in the training and test set, endoscopic activity classes were balanced in the validation set.

The neural network model was developed using the baseline endoscopic activity and all four selected variables as inputs to predict whether a UC patient will have an active or inactive endoscopic disease at one year, under the same therapeutic regimen. Model performance metrics are shown in Table 2. ROC curves proving model performance on the train, test, and validation sets are shown in Figure 2.

Table 2. The classifier's performance metrics.

\begin{tabular}{|c|c|c|c|}
\hline & Train Set & Test Set & Validation Set \\
\hline & Predictions & Predictions & Predictions \\
\hline Actual & Remission Activity & Remission Activity & Remission Activity \\
\hline Remission & 27 & 1 & 0 \\
\hline Activity & 7 & 3 & 2 \\
\hline $\mathrm{ACC}$ & $85 \%$ & $90 \%$ & $100 \%$ \\
\hline $95 \% \mathrm{CI}$ & $(0.70,0.94)$ & $(0.56,0.99)$ & $(0.48,1.00)$ \\
\hline$p$ value & $<0.001$ & $<0.001$ & $<0.001$ \\
\hline SE & $82 \%$ & $100 \%$ & $100 \%$ \\
\hline $\mathrm{SP}$ & $100 \%$ & $75 \%$ & $100 \%$ \\
\hline PPV & $100 \%$ & $86 \%$ & $100 \%$ \\
\hline NPV & $54 \%$ & $100 \%$ & $100 \%$ \\
\hline AUC & 0.91 & 0.92 & 1.00 \\
\hline
\end{tabular}

ACC (Accuracy); CI (Confidence Intervals); AUC (Area under the receiver operating characteristic curve); SE (Sensitivity); SP (Specificity), PPV (Positive predictive value); NPV (Negative predictive value). 


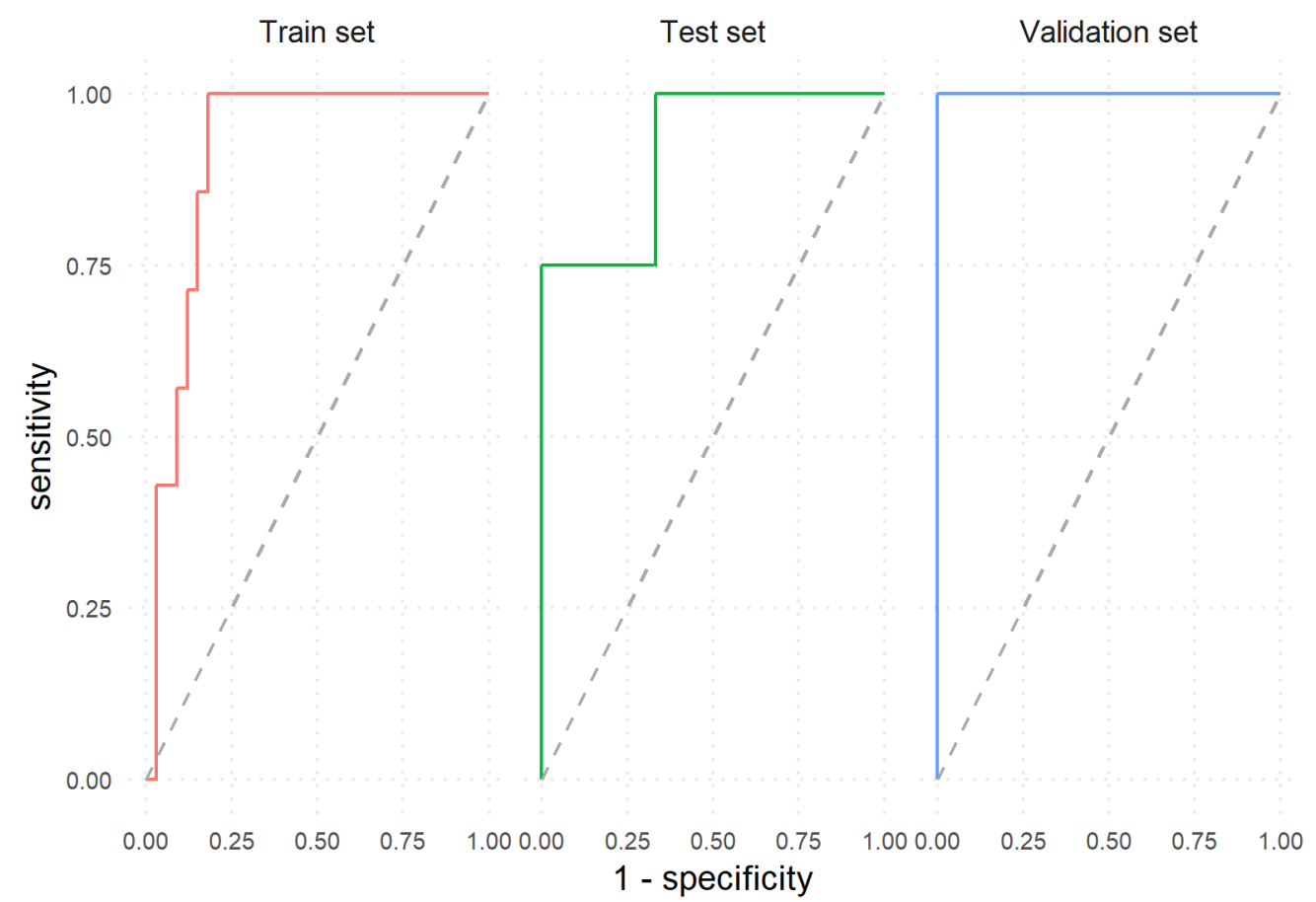

Figure 2. Classifier's performance to predict endoscopic remission vs. relapse at one year.

\section{Discussion}

Our study is the first neural network developed for UC patients treated with anti-TNF agents for predicting endoscopic disease activity and risk of relapse at one year using standard baseline parameters. We demonstrated that it is possible to accurately predict anti-TNF response at one year in UC patients using machine learning methods. Present-day guidelines [25-27] indicate that the best future activity prediction is based on current endoscopic activity. Our solution brings a new perspective by incorporating additional parameters besides baseline endoscopy to predict future disease outcomes.

Measuring endoscopic disease activity is the "gold" standard for disease monitoring in UC according to current guidelines [25-27]. Indeed, strong evidence shows that targeting endoscopic healing is superior to tailing only clinical remission (concerning relapse rates, hospitalization rates, and the need for surgery) [28]. The advent of biological therapy in UC paved the way for achieving more profound, subclinical remission degrees (mucosal and histological healing). However, the inadequate response to biologics delays the disease's resolution, exposes patients to unnecessary toxic drug effects, and wastes medical resources. Therefore, the growing interest in monitoring the therapy aims to identify appropriate end-points for successful treatment and timely discontinue or switch the therapy in those likely to relapse or unlikely to respond [29]. The proper therapeutic decision is essential, even more so as inopportune discontinuation may trigger the development of anti-drug antibodies that can lead to future response loss [30].

We aimed to develop a machine learning tool capable of predicting the endoscopic disease activity at one year. Comparing the baseline activity with the predicted one and acknowledging the risk of relapse, the clinician may decide whether increasing the dose, switching to other biologic agents, or discontinuing the therapy is the most appropriate decision. Our model achieved an ACC of $85 \%$ with $82 \%$ SE, $100 \%$ SP and an AUC of 0.91 on the trainset. On the test set, the classifier obtained an excellent performance with an ACC of $90 \%$, SE of $100 \%$, SP of $75 \%$, and AUC of 0.92 . On the validation set, the model predictions achieved the maximum performance with a $100 \%$ ACC, SE, and SP and an AUC of 1.

A few other studies aimed to predict endoscopic remission at one year. One paper used faecal calprotectin (FC) assay measured after the induction of anti-TNF therapy (infliximab) to predict the mucosal healing after one year of treatment [31]. A cut-off of $\leq 121 \mu \mathrm{g} / \mathrm{g}$ used for the post-induction 
faecal calprotectin achieved $70 \% \mathrm{SE}$ and $70 \% \mathrm{SP}$ for predicting mucosal healing at one year in 50 patients with UC colonic or ileal-colonic Crohn's disease. Our model obtained higher performance metrics on all tested sets.

A particular aspect worth mentioning is that, in our study, baseline CRP levels differed significantly in patients developing an active disease versus patients in endoscopic remission at one year. Our result is consistent with the findings of other studies. CRP was shown to be a clinically relevant biomarker of response to infliximab [32] and an independent predictor of colectomy-free survival in patients treated with infliximab [33].

\section{Limitations and Future Perspectives}

Firstly, our dataset's minimal size and the fact that the independent validation set is from the same centre entails rigorous external validation with data from other centres. Secondly, the imbalanced distribution of endoscopic activity classes at one year predisposes to calculation biases, although the SMOTE function in $\mathrm{R}$ was used to reduce these biases significantly. Thirdly, the retrospective nature of our study may introduce further bias.

In the future, these drawbacks could be overcome by employing prospective studies on broader, more diverse, and comprehensive datasets in a centre with greater accessibility that would permit organizing a cohort with a balanced distribution of endoscopic activity classes both at baseline and at one year. The next trials would improve models' performance using different ML algorithms as our patient's database extends.

\section{Conclusions}

Our proposed ML solution proved to accurately predict disease activity at one year in UC patients treated with anti-TNF agents using routinely available clinical parameters. Acknowledging the risk of relapse could lead to increasing the dose or switching to other biological agents. After rigorous validation on large, external datasets, our ML approach could significantly impact clinical practice by helping the physician decide on the most appropriate therapeutic option concerning the management of anti-TNF biologics.

Author Contributions: Conceptualization, I.V.P. and C.C.P.; methodology, I.V.P. and A.B.; software, I.V.P.; validation, I.V.P. and A.B.; formal analysis, I.V.P. and A.B.; investigation, I.V.P.; resources, C.M. and C.C.P.; data curation, I.V.P.; writing—original draft preparation, I.V.P. and A.B.; writing-review and editing, A.B. and C.M.; supervision, A.B. and C.C.P. All authors have read and agreed to the published version of the manuscript.

Funding: This research received no external funding.

Conflicts of Interest: The authors declare no conflict of interest.

\section{References}

1. Danese, S.; Roda, G.; Peyrin-Biroulet, L. Evolving Therapeutic Goals in Ulcerative Colitis: Towards Disease Clearance. Nat. Rev. Gastroenterol. Hepatol. 2020, 17, 1-2. [CrossRef]

2. Paramsothy, S.; Rosenstein, A.K.; Mehandru, S.; Colombel, J.-F. The current state of the art for biological therapies and new small molecules in inflammatory bowel disease. Mucosal. Immunol. 2018, 11, 1558-1570. [CrossRef]

3. Park, S.C.; Jeen, Y.T. Current and emerging biologics for ulcerative colitis. Gut Liver 2015, 9, 18-27. [CrossRef]

4. Holdam, A.S.; Bager, P.; Dahlerup, J.F. Biological therapy increases the health-related quality of life in patients with inflammatory bowel disease in a clinical setting. Scand. J. Gastroenterol. 2016, 51, 706-711. [CrossRef]

5. D'Haens, G. Risks and benefits of biologic therapy for inflammatory bowel diseases. Gut 2007, 56, 725-732. [CrossRef]

6. Rubin, D.T. When Should Therapy for Inflammatory Bowel Disease Be Stopped? Gastroenterol. Hepatol. 2015, $11,400-402$.

7. Moss, A.C. Optimizing the use of biological therapy in patients with inflammatory bowel disease. Gastroenterol. Rep. 2015, 3, 63-68. [CrossRef] 
8. Waljee, A.K.; Chaisidhivej, N.; Saini, S.D.; Higgins, P.D.R. De-escalation of IBD Therapy: When, Who, and How? Crohns Colitis 360 2019, 1, otz008. [CrossRef]

9. Ha, C.; Ullman, T.A.; Siegel, C.A.; Kornbluth, A. Patients enrolled in randomized controlled trials do not represent the inflammatory bowel disease patient population. Clin. Gastroenterol. Hepatol. 2012, 10, 1002-1007. [CrossRef]

10. Ben-Horin, S.; Kopylov, U.; Chowers, Y. Optimizing anti-TNF treatments in inflammatory bowel disease. Autoimmun. Rev. 2014, 13, 24-30. [CrossRef]

11. Lopetuso, L.R.; Gerardi, V.; Papa, V.; Scaldaferri, F.; Rapaccini, G.L.; Gasbarrini, A.; Papa, A. Can We Predict the Efficacy of Anti-TNF- $\alpha$ Agents? Int. J. Mol. Sci. 2017, 18, 1973. [CrossRef]

12. Louis, E. Tailoring Biologic or Immunomodulator Treatment Withdrawal in Inflammatory Bowel Disease. Front. Med. 2019, 6, 302. [CrossRef]

13. Li, J.; Qian, J.M. Artificial intelligence in inflammatory bowel disease: Current status and opportunities. Chin. Med. J. 2020, 133, 757-759. [CrossRef]

14. Cogan, T.; Cogan, M.; Tamil, L. MAPGI: Accurate identification of anatomical landmarks and diseased tissue in gastrointestinal tract using deep learning. Comput. Biol. Med. 2019, 111, 103351. [CrossRef]

15. Maeda, Y.; Kudo, S.; Ogata, N.; Misawa, M.; Mori, Y.; Mori, K.; Ohtsuka, K. Can artificial intelligence help to detect dysplasia in patients with ulcerative colitis? Endoscopy 2020. [CrossRef]

16. Takenaka, K.; Ohtsuka, K.; Fujii, T.; Negi, M.; Suzuki, K.; Shimizu, H.; Oshima, S.; Akiyama, S.; Motobayashi, M.; Nagahori, M.; et al. Development and Validation of a Deep Neural Network for Accurate Evaluation of Endoscopic Images From Patients With Ulcerative Colitis. Gastroenterology 2020, 158, 2150-2157. [CrossRef]

17. Forbes, J.D.; Chen, C.-Y.; Knox, N.C.; Marrie, R.-A.; El-Gabalawy, H.; de Kievit, T.; Alfa, M.; Bernstein, C.N.; van Domselaar, G. A comparative study of the gut microbiota in immune-mediated inflammatory diseases-does a common dysbiosis exist? Microbiome 2018, 6, 221. [CrossRef]

18. Mossotto, E.; Ashton, J.J.; Coelho, T.; Beattie, R.M.; MacArthur, B.D.; Ennis, S. Classification of Paediatric Inflammatory Bowel Disease using Machine Learning. Sci. Rep. 2017, 7, 2427. [CrossRef]

19. Biasci, D.; Jc, L.; Nm, N. A blood-based prognostic biomarker in IBD. Gut 2019, 68, 1386-1395. [CrossRef]

20. Zarringhalam, K.; Enayetallah, A.; Reddy, P.; Ziemek, D. Robust clinical outcome prediction based on Bayesian analysis of transcriptional profiles and prior causal networks. Bioinformatics 2014, 30, i69-i77. [CrossRef]

21. Douglas, G.M.; Hansen, R.; Jones, C.M.A.; Dunn, K.A.; Comeau, A.M.; Bielawski, J.P.; Tayler, R.; El-Omar, E.M.; Russell, R.K.; Hold, G.L.; et al. Multi-omics differentially classify disease state and treatment outcome in pediatric Crohn's disease. Microbiome 2018, 6, 13. [CrossRef] [PubMed]

22. Waljee, A.K.; Liu, B.; Sauder, K.; Zhu, J.; Govani, S.M.; Stidham, R.W.; Higgins, P.D.R. Predicting corticosteroid-free endoscopic remission with vedolizumab in ulcerative colitis. Aliment. Pharmacol. Ther. 2018, 47, 763-772. [CrossRef] [PubMed]

23. Hardalaç, F.; Başaranoğlu, M.; Yüksel, M.; Kutbay, U.; Kaplan, M.; Demirbağ, A.E.; Coşkun, O.; Aksoy, A.; Gangarapu, V.; Örmeci, N.; et al. The rate of mucosal healing by azathioprine therapy and prediction by artificial systems. Turk. J. Gastroenterol. 2015, 26, 315-321. [CrossRef] [PubMed]

24. Maaser, C.; Sturm, A.; Vavricka, S.R.; Kucharzik, T.; Fiorino, G.; Annese, V.; Calabrese, E.; Baumgart, D.C.; Bettenworth, D.; Nunes, P.B.; et al. ECCO-ESGAR Guideline for Diagnostic Assessment in IBD Part 1: Initial diagnosis, monitoring of known IBD, detection of complications. J. Crohns Colitis 2019, 13, 144-164. [CrossRef] [PubMed]

25. Annese, V.; Daperno, M.; Rutter, M.D.; Amiot, A.; Bossuyt, P.; East, J.; Ferrante, M.; Götz, M.; Katsanos, K.H.; Kießlich, R.; et al. European evidence based consensus for endoscopy in inflammatory bowel disease. J. Crohns Colitis 2013, 7, 982-1018. [CrossRef]

26. Magro, F.; Gionchetti, P.; Eliakim, R.; Ardizzone, S.; Armuzzi, A.; Acosta, M.B.; Burisch, J.; Gecse, K.B.; Hart, A.L.; Hindryckx, P.; et al. Third European Evidence-based Consensus on Diagnosis and Management of Ulcerative Colitis. Part 1: Definitions, Diagnosis, Extra-intestinal Manifestations, Pregnancy, Cancer Surveillance, Surgery, and Ileo-anal Pouch Disorders. J. Crohns Colitis 2017, 11, 649-670. [CrossRef]

27. Lamb, C.A.; Kennedy, N.A.; Raine, T.; Hendy, P.A.; Smith, P.J.; Limdi, J.K.; Hayee, B.; Lomer, M.C.E.; Parkes, G.C.; Selinger, C.; et al. British Society of Gastroenterology consensus guidelines on the management of inflammatory bowel disease in adults. Gut 2019, 68 (Suppl. S3), s1-s106. [CrossRef] 
28. Zallot, C.; Peyrin-Biroulet, L. Deep remission in inflammatory bowel disease: Looking beyond symptoms. Curr. Gastroenterol. Rep. 2013, 15, 315. [CrossRef]

29. Ben-Horin, S.; Mao, R.; Chen, M. Optimizing biologic treatment in IBD: Objective measures, but when, how and how often? BMC Gastroenterol. 2015, 15, 178. [CrossRef]

30. Kothari, M.M.; Nguyen, D.L.; Parekh, N.K. Strategies for overcoming anti-tumor necrosis factor drug antibodies in inflammatory bowel disease: Case series and review of literature. World J. Gastrointest. Pharmacol. Ther. 2017, 8, 155-161. [CrossRef]

31. Guidi, L.; Marzo, M.; Andrisani, G.; Felice, C.; Pugliese, D.; Mocci, G.; Nardone, O.; de Vitis, I.; Papa, A.; Rapaccini, G.; et al. Faecal calprotectin assay after induction with anti-Tumour Necrosis Factor $\alpha$ agents in inflammatory bowel disease: Prediction of clinical response and mucosal healing at one year. Dig. Liver Dis. 2014, 46, 974-979. [CrossRef] [PubMed]

32. Iwasa, R.; Yamada, A.; Sono, K.; Furukawa, R.; Takeuchi, K.; Suzuki, Y. C-reactive protein level at 2 weeks following initiation of infliximab induction therapy predicts outcomes in patients with ulcerative colitis: A 3 year follow-up study. BMC Gastroenterol. 2015, 15, 103. [CrossRef] [PubMed]

33. Arias, M.T.; Casteele, N.V.; Vermeire, S.; de Buck van Overstraeten, A.; Billiet, T.; Baert, F.; Wolthuis, A.; van Assche, G.; Noman, M.; Hoffman, I.; et al. A panel to predict long-term outcome of infliximab therapy for patients with ulcerative colitis. Clin. Gastroenterol. Hepatol. 2015, 13, 531-538. [CrossRef] [PubMed]

Publisher's Note: MDPI stays neutral with regard to jurisdictional claims in published maps and institutional affiliations. 